\title{
Research on New Energy Recovery Retarder
}

\author{
Haiting Zhang, ${ }^{1, a}$, Yuanqi Cai ${ }^{1, a}$, Jianbo Cao ${ }^{1, b *}$, Jianxia Cao ${ }^{2, b}$,Tianfeng Zhao ${ }^{3, b}$, \\ Yanger Shi ${ }^{1, a}$, Shiju $\mathrm{E}^{1, \mathrm{~b}}$ and Yi Wang ${ }^{1, a}$ \\ ${ }^{1}$ College of Engineering, Zhejiang Normal University, Jinhua, Zhejiang 321004, P.R. China \\ ${ }^{2}$ Jinan Jindingnuo Pump Co., Ltd, Jinan, Shandong 250100, P.R. China \\ ${ }^{3}$ Library, Zhejiang Normal University, Jinhua, Zhejiang 321004, P.R. China

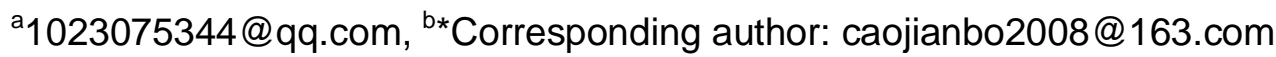

\section{Keywords: Energy recovery, Retarder, Energy saving, Brushless DC motor}

Abstract. Car retarder is an auxiliary braking device. It is very important to improve the driving safety of the automobile. At present, the domestic and foreign research car retarder is mainly based on eddy current, permanent magnet type and hydraulic. Although the traditional retarder can play the auxiliary braking effect, but kineticing energy into heat energy to the air is a waste of energy. In order to solve this problem, this paper designs a kind of energy recovery retarder. Through the research and study of mechanical structure of the permanent magnet brushless DC motor, and analyzing the structure of the existing retarder, the assembly drawing of mechanical structure of energy recovery retarder and three-dimensional model are set up, and the installation of the retarder braking in the structure is put forward. Based on the mathematical model of permanent magnetic brushless DC motor, and after researching the working principle of energy recovery retarder, the results of this study has great value for the car's safety and energy saving and environmental protection.

\section{Introduction}

Car retarder is an auxiliary braking device. Its function is to produce brake moment. Performance of retarder directly affects the vehicle braking stability and speed. To improve the driving safety of the automobile plays a very important role.

From the beginning of twentieth Century 90 years, China has entered a period of rapid development of highway construction, as of the end of 2011, the total highway mileage has been $4000000 \mathrm{~km}$. The highway starts from zero, after more than 20 years of development, by the end of 2011, the national highway mileage has reached 85000 kilometers, ranking second in the world [1]. China is a mountainous country, mountains area (including the mountains and hills and rugged plateau) accounted for about $70 \%$, the formation of the mountainous terrain features. Lower grade roads, mountain road accounted for a large proportion. The conditions of Fujian, Jiangxi, Yunnan, Sichuan and Shaanxi area extremely have harsh road [2].

Along with the progress of automobile technology, automobile engine power increased year by year. When long time friction brake, bus and heavy truck will produce a great quantity of heat, which makes the brake shoes and brake wheel's temperature rise, leading to a low coefficient of friction, braking efficiency braking ability decreased, even disappeared, resulting in serious consequences [3]. Large tonnage vehicles traffic accidents are continue to occur. The more stringent requirements of the automobile braking device is put forward [4].

In this case, it requests the automobile industry to produce more suitable for highway and city running cars, these vehicles will not only has the characteristics of high power, high comfort, and in particular a very high safety [5]-[7]. So the vehicle auxiliary braking system (device) of the retarder gradually gets used in domestic. It consumes the most energy in the vehicle before brake, enhances the braking efficiency, improves the safety performance of vehicles.

At present, the research is still concentrated in the retarder braking characteristics and control method, the new retarder (especially for energy recovery retarder) design and research are involved less. And although the traditional retarder can play auxiliary braking effect, the vehicle kinetic energy into heat energy to the air which is a waste of energy. In order to solve this problem, this paper designs a kind of energy recovery retarder. 


\section{Working Principle of Energy Recovery Retarder System}

Energy recovery retarder system consists of a permanent magnet brushless DC motor and its controller control. The controller's main function is the power converter, signal acquisition and state detection. Energy recovery retarder system hardware mainly includes energy storage device, the inverter circuit, power module drive and protection circuit, current and voltage detection circuit, position detection circuit and the power management module. The hardware principle diagram is shown in Fig. 1.

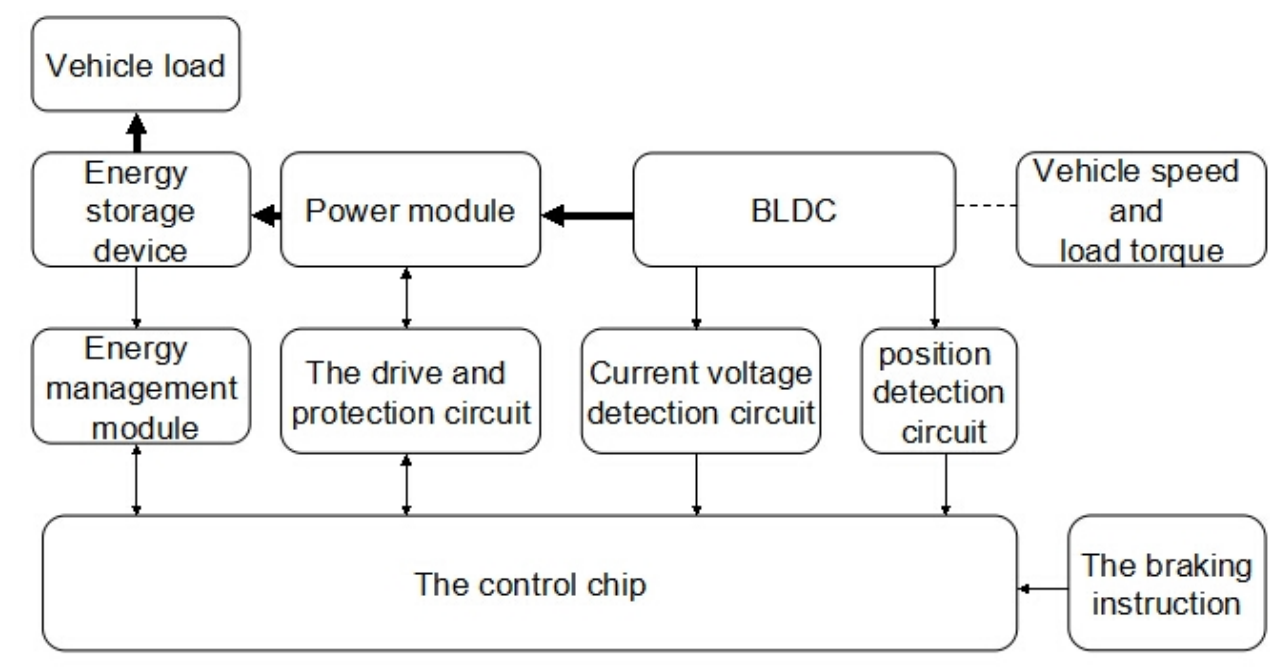

Fig. 1 Energy recovery retarder hardware principle diagram

As the diagram shown in Fig. 1 depicts the relationship between the various parts of the energy recovery retarder, the thick arrow to indicate the direction of energy flow, thin arrows represent the direction of transmission signal. When braking, the kinetic energy of the vehicle under the effect of the retarder is converted to electrical energy by the power module which stores the electric energy to the energy storage device. Energy management module is used to detect the vehicle battery state of charge, and the temperature of the battery, to send signals to control the chip, waiting to be processed; power module drive circuit is used to determine the position of the power; current and voltage detection circuit to the winding current and voltage of the permanent magnetic measurement brushless motor position signal feedback to the control chip and the position sensor was processed to form a closed-loop control.

\section{Mechanical Structure Design of Energy Recovery Retarder}

Structure Description. Retarder is installed in the gear box. The main reducer is between the first axis of rotation. The retarder and gearbox are connected through the coupling, as shown in Fig. 2. Retarder consists of a stator assembly and the rotor assembly. The stator assembly is composed of a plurality of coils. A rotor assembly is composed of magnetic cylinder.

Two Dimensional Drawing and Assembly Drawing. Through the AotoCAD 2D part drawings and assembly drawings design of energy recovery retarder, its body size is according to the size of the market of the general retarder. The size and tolerance of parts arrangement is according to the requirements of assembly, torque and size, as shown in Fig. 3 to Fig. 5.

Three Dimensional Diagram of Energy Recovery Retarder. Through Solidworks 3D assembly drawing design of energy recovery retarder is shown in Fig. 6. 


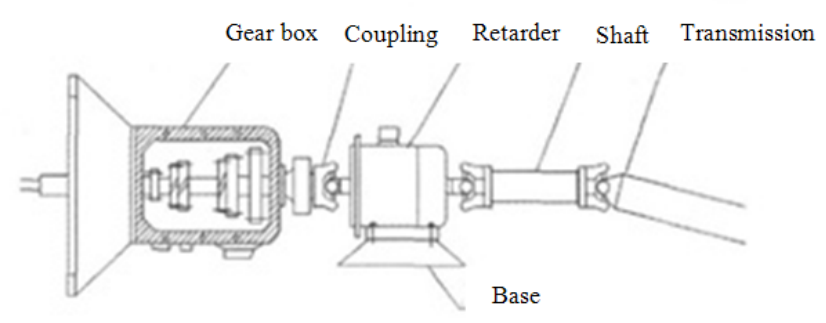

Fig. 2 Energy recovery retarder connection diagram
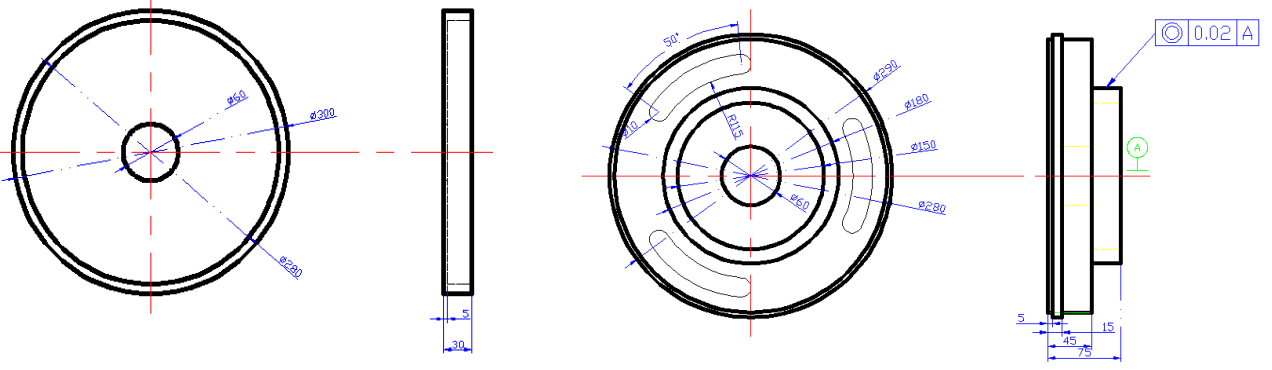

Fig.3 Retarder end cap

Fig. 4 Retarder bearing seat
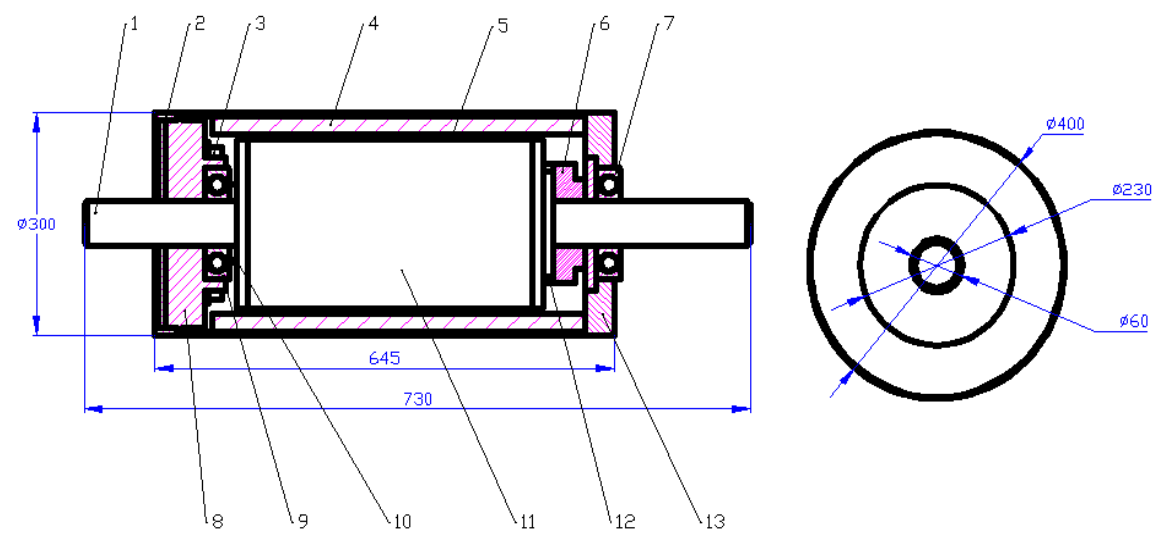

1-Transmission shaft 2-End cap 3-Holzer sensor 4-Stack 5-Coil 6-Ring

7、9-Rolling bearing 8-Bearing seat 10-Washer 11-Magnetic cylinder 12-Spring washer 13-Shell

Fig. 5 Energy recovery retarder assembly drawing

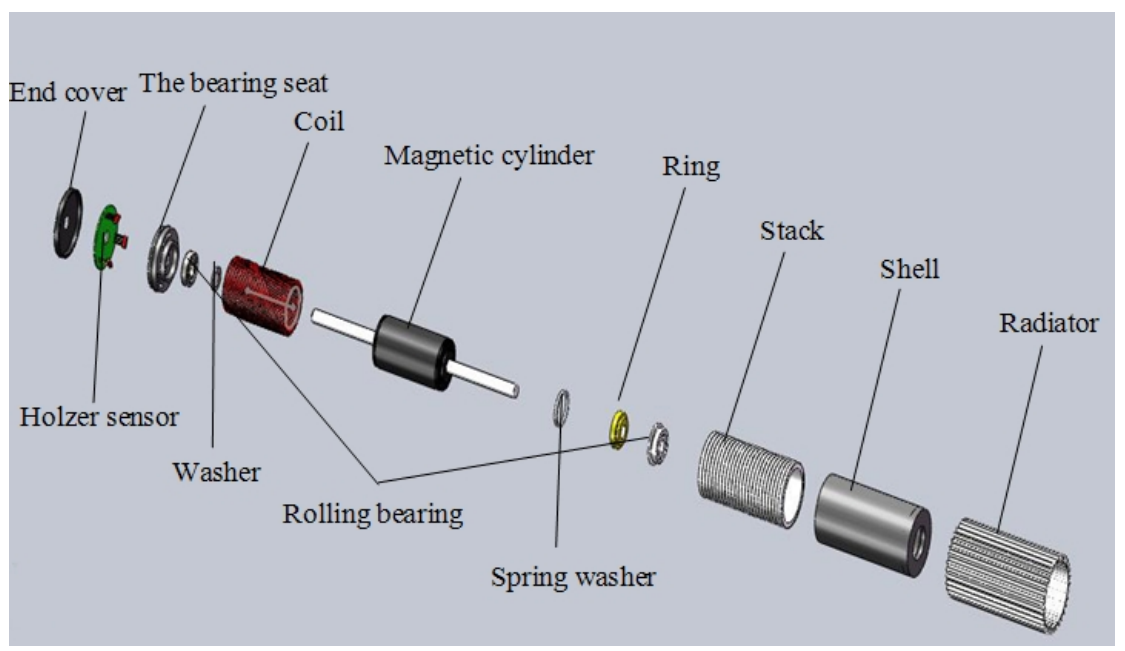

Fig. 6 Energy recovery retarder internal structure 


\section{Study on Principle and Mathematical Model of Energy Recovery}

The fundamental principle of energy recovery retarder energy recovery is a motor energy feedback braking. According to the different purposes of the motor brake, the brake can be divided into two kinds of braking and emergency braking. Braking motor rotation is still in the state, but in the opposite direction of motor electromagnetic torque and rotation state, by gradually slow down until the stop. Emergency brake motor quickly stop, need to immediately stop the motor rotation of the rotor, the braking torque large, usually by friction to achieve. The retarder using brushless motor and brake pad and brake. In the process of emergency braking, the kinetic energy of the system is transformed into a part of the friction heat, is a kind of energy consumption, the other part of stored energy into electricity. The braking deceleration can be achieved through the electric braking mode.

\section{Conclusion}

In this paper, mechanical mechanism of energy recovery retarder has been researched and designed. Retarder is installed in the gear box and the main reducer between the first axis of rotation. Retarder and gearbox are connected through the coupling. Retarder consists of a stator assembly and the rotor assembly. The stator assembly is composed of a plurality of coils, a rotor assembly is composed of magnetic cylinder. Through the platform of 2D assembly drawing, 3D model, structure of the retarder has the overall design. Energy recovery retarder has broad development prospects in the energy recovery, the market will have great potential.

\section{Acknowledgement}

The work was supported by the National Natural Science Foundation of China (No. 51377146, 51407162), Zhejiang Provincial Natural Science Foundation of China (No. LQ13E050009), Zhejiang Provincial Postdoctoral Research Project Merit-based Funding (No. BSH1301008), the National Innovation and Entrepreneurship Training Program of University Students (No. 201410345008), the 2013 Foundation of Education Department of Zhejiang Province of China (No. Y201329556).

\section{References}

[1] J. Li: Technology Wind, (2012) No. 8, pp. 146.

[2] X.B. Zhao: Research on Electromagnetic Properties and Braking Performance of Permanent Magnet Eddy Current Retarder (Nanjing Agricultural University, China 2009).

[3] P. Sun, R. Song and X.H. Wang: Safety and Environmental Engineering, Vol. 14 (2007) No. 2, pp. 97-100.

[4] L.Z. Ye: Design Theory and Experimental Study on Automobile Permanent Magnet Retarder (Beijing University of Technology, China 2012).

[5] Z.Y. Yang: Design of Energy Recovery Electric Eddy Current Retarder (Nanjing University of Science and Technology, China 2006).

[6] D.P. Ma, Y. Li and Q.O. Liang: Journal of Zhejiang Normal University (Natural Sciences), Vol. 38 (2015) No. 1, pp. 116-120.

[7] V. Bist and B. Singh: Electric Power Systems Research, (2013) No. 98, pp. 11-18. 